\title{
Mesure de l'intensité et de la sélectivité du bronzage zooplanctonique diurne et nocturne sur le peuplement physoplanctonique de la retenue Al Massira (Maroc) Estimation of the diel zooplankton grazing selectivity on the phytoplankton community of the Al Massira Reservoir (Morocco)
}

\author{
M. Moncef, M. Malki, A. Fahde et J. Devaux
}

Volume 7, numéro 1, 1994

URI : https://id.erudit.org/iderudit/705186ar

DOI : https://doi.org/10.7202/705186ar

Aller au sommaire du numéro

\section{Éditeur(s)}

Université du Québec - INRS-Eau, Terre et Environnement (INRS-ETE)

ISSN

0992-7158 (imprimé)

1718-8598 (numérique)

Découvrir la revue

Citer cet article

Moncef, M., Malki, M., Fahde, A. \& Devaux, J. (1994). Mesure de l'intensité et de la sélectivité du bronzage zooplanctonique diurne et nocturne sur le peuplement physoplanctonique de la retenue $\mathrm{Al}$ Massira (Maroc). Revue des sciences de l'eau / Journal of Water Science, 7(1), 3-22.

https://doi.org/10.7202/705186ar

\section{Résumé de l'article}

La retenue de barrage Al Massira (Maroc) constitue une pièce maîtresse dans le plan d'aménagement du bassin versant de l'Oum Er Rbia. Elle se situe dans la province de Settat, à $120 \mathrm{~km}$ au sud-est de Casablanca. La mesure de l'intensité du broutage zooplanctonique sur le peuplement phytoplanctonique a été réalisée à trois périodes différentes; le 20 mars 1992 d'une part et les 27 et 28 mai 1992 d'autre part, avec respectivement des mesures de jour et de nuit . Pour cela, une analyse de l'évolution des densités phytoplanctoniques au sein d'enceintes immergées in situ à 5 et $15 \mathrm{~m}$ pendant 8 heures, en absence (enceintes témoins) et en présence (enceintes expérimentales) de zooplancton, a été réalisée. Les résultats obtenus montrent que:

- La technique adoptée est performante dans la mesure où les différences entre les densités algales entre les temps initial et final sont en général significatives dans les deux enceintes.

- Conformément aux données de la littérature, le broutage apparaît bien comme sélectif; de nombreuses espèces n'apparaissant pas être consommées.

- La sélection effectuée par le zooplancton est principalement basée sur la taille des cellules. Toutefois, à tailles égales, certaines espèces phytoplanctoniques semblent être consommées préférentiellement par les copépodes.

- Seules les espèces algales présentant un développement important dans les enceintes témoins sont soumises à la prédation. 


\title{
Mesure de l'intensité et de la sélectivité du broutage zooplanctonique diurne et nocturne sur le peuplement phytoplanctonique de la retenue Al Massira (Maroc)
}

\author{
Estimation of the diel zooplankton grazing selectivity \\ on the phytoplankton community \\ of the Al Massira Reservoir (Morocco)
}

Reçu le 23 octobre 1992, accepté le 15 octobre 1993*.

\section{SUMMARY}

The Al Massira reservoir, located in the district of Settat $120 \mathrm{~km}$ south-east of Casablanca, is of great importance notably at the level of the management of the Oum Er Rbia River-catchment basin.

Several grazing experiments were performed to assess the intensity of phytoplankton predation by zooplankton species, on 20 March 1992, 27 and 28 May 1992, respectively, over the day and the night. Two enclosures were used and incubated in situ at 5 and $15 \mathrm{~m}$ over 8 hours. The first enclosure consisted of a mixture of several phytoplankton and zooplankton species. The second was free of zooplancton.

The results clearly demonstrated that the technique used in this work seemed to be effective in that a significant difference between the initial and final numbers of phytoplankton species incubated in the enclosure was detected. In addition, we confirmed several literature reports relative to the selective characteristics of herbivore predation on algae: large phytoplankton (e.g., Peridinium cinctum, Ceratium hirundinella and Staurastrum pingue) were not affected. The grazing pressure depended on the size of preys and on the exudates released by the physiologically active small phytoplankton species (e.g., Oocystis crassa, Cyclotella sp.) evolving in the enclosures. Among small preys, only species with high growth rate (estimed in the control flask, e.g., Cosmarium sp. and Tetraedron sp.) were subjected to the zooplankton predation. The copepod nightgrazing was lower than that recorder during the day, probably due to a decrease in their ability to detect preys in the dark.

Our result contradict several results obtained by the method of HANEY (1971) according to which grazing increases during the night.

1. Université Chouaib Doukkali, Faculté des sciences. El Jadica (Maroc).

2. Université Hassan II, Faculté des sciences I. Casablanca (Maroc).

3. Université Blaise Pascal de Clermont Ferrand II. Groupe de Zoologie et Protistologie, 63177 Aubière Cedex (France).

* Les commentaires seront reçus jusqu'au 31 octobre 1994. 
Two assumptions may be proposed to explain such a discrepancy:

- The zooplankton community of the Al Massira Reservoir was quantitatively dominated by the Calanoid Neolovenula alluaudi. Yet, according to HANEY and HALL (1975), these Copepods showed a slight diel grazing change compared to that of Cladocera. It seemed likely that these temporal variabilities were not statistically significant but rather inherent to the overall internal fluctuation of the cell metabolism.

- The HANEY (1971) method allows estimation of the water volume per unit time, which transits along the digestive tract of zooplankters. Nevertheless, this technique was mostly performed in markedly stratified natural lakes. From a strictly physiological point of view, and as demonstrated by MC LAREN (1963), organisms which evolved in deep layers (low temperature) became metabolically mone active when they reached superficial layers (increased temperature). Clearly, this increase in the cell metabolism abilities is translated into a higher volume of water passing through the digestive tract, but not necessarily into an increase in ingested phytoplankton preys. Our results went along with the forementioned interpretation since we clearly demonstrated an optimal Copepoda-grazing selectivity. However, the selection of preys would not imply a direct relationship between the ingested volume of water and energetic inputs.

Key-words : reservoir, zooplankton, phytoplankton, grazing.

La retenue de barrage Al Massira (Maroc) constitue une pièce maîtresse dans le plan d'aménagement du bassin versant de l'Oum Er Rbia. Elle se situe dans la province de Settat, à $120 \mathrm{~km}$ au sudest de Casablanca. La mesure de l'intensité du broutage zooplanctonique sur le peuplement phytoplanctonique a été réalisée à trois périodes différentes ; le 20 mars 1992 d'une part et les 27 et 28 mai 1992 d'autre part, avec respectivement des mesures de jour et de nuit. Pour cela, une analyse de l'évolution des densités phytoplanctoniques au sein d'enceintes immergées in situ à 5 et $15 \mathrm{~m}$ pendant 8 heures, en absence (enceintes témoins) et en présence (enceintes expérimentales) de zooplancton, a été réalisée. Les résultats obtenus montrent que :

- La technique adoptée est performante dans la mesure où les différences entre les densités algales entre les temps initial et final sont en général significatives dans les deux enceintes.

- Conformément aux données de la littérature, le broutage apparât bien conme sélectif ; de nombreuses espèces n'apparaissant pas être consommées.

- La sélection effectuée par le zooplancton est principalement basée sur la taille des cellules. Toutefois, à tailles égales, certaines espèces phytoplanctoniques semblent être consommées préférentiellement par les copépodes.

- Seules les espèces algales présentant un développement important dans les enceintes témoins sont soumises à la prédation.

Mots clés : retenue, zooplancton, phytoplancton, broutage. 


\section{1 - INTRODUCTION}

La prédation par le zooplancton constitue avec la lyse cellulaire et la sédimentation, l'une des causes majeures de disparition du phytoplancton au sein des écosystèmes lacustres.

II s'agit, de plus, du flux de transfert de la matière organique produite par voie photosynthétique, dans le réseau alimentaire.

L'analyse de ce processus àpparaît donc comme essentielle dans la perspective d'une compréhension globale du fonctionnement des biocénoses limniques. Sa quantification se heurte toutefois à de nombreux problèmes d'ordre méthodologique. Trois grands types de méthodes sont actuellement utilisés :

- Mesure, à partir d'incubation en enceintes immergées in situ, de l'évolution de cellules phytoplanctoniques après ajout au sein du peuplement naturel d'une population préalablement marquée au ${ }^{14} \mathrm{C}$ (HANEY, 1971). Bien que très sensible, cette méthode ne fournit qu'une quantification du volume d'eau transitant dans le tube digestif des zooplanctontes.

- Recherche dans le contenu intestinal des animaux, de molécules organiques caractéristiques des différentes classes d'algues planctoniques: acides gras (BOURDIER et AMBLARD, 1989 ; BOURDIER, 1989), pigments (MACKAS et BORHER, 1976 ; QUIBLIER et al., 1991). Bien que très prometteuses, ces techniques qui ne nécessitent pas de mise en incubation de peuplement, n'autorisent pas encore une estimation quantitative de la prédation.

- Mesure, par dénombrements cellulaires après incubation en enceintes, de l'évolution quantitative des populations phytoplanctoniques naturelles, en absence et en présence de zooplancton (PORTER,1972). C'est ce dernier type de technique que nous avons retenu. Bien que sa mise en œurre soit relativement longue, cette méthode présente l'avantage de pouvoir juger de l'éventuelle sélectivité du broutage.

Ainsi trois expériences de broutage ont été réalisées :

- La première, effectuée le 20 mars 1992, avait un double objectif :

D'une part s'assurer de la fiabilité de la méthode choisie.

D'autre part mesurer l'intensité et la sélectivité du broutage exercé par le zooplancton sur le peuplement phytoplanctonique de la retenue.

- Les deux autres expériences ont été réalisées les 27 et 28 mai 1992 respectivement le jour et la nuit dans le but d'évaluer la variabilité nycthémérale du broutage des populations algales par les copépodes.

La présente étude s'intègre dans le cadre d'un programme pluridisciplinaire débuté en 1989 sur la retenue Al Massira afin de modéliser le fonctionnement de cet écosystème situé en climat aride. 


\section{2 - MATÉRIEL ET MÉTHODES}

\section{2..1 Site d'éturdes}

La renenue A la pièce maîtresse du Jlan d'aménajemerl du bizssin wrarsant de lcued E:- Rbia. Elle sio situe dans la fróince cie Settiat à $T 20 \mathrm{~km}$ al $\leqq i d-\epsilon$ C.zsablanca. Less caractéristigues frincipales de cette retenute serri do par le tableau 1

Tableau in Caractéristiques géomorphologiques de la retemue Al Massite.

Table 1

Mormhometric characteristics of Ai! Massira Reservoir.

\begin{tabular}{|c|c|}
\hline Paramettres & Valmurs \\
\hline Surfface du brassin versiant & $28 \mathrm{~s} \$ 0 \mathrm{~km}{ }^{2}$ \\
\hline Prrotondeur miaximale & $40 \mathrm{~m}$ \\
\hline Profondeur Imıyenne à lấ côte for tale & $20 \mathrm{~m}$ \\
\hline Longeur & $30 \mathrm{~km}$ \\
\hline Larrgeur & $10 \mathrm{~km}$ \\
\hline Voume au niweau nomal & $2800 \cdot 10^{6} \mathrm{~m}^{2}$ \\
\hline Pluviométrie moyenne & $302 \pi \mathrm{mm}$ \\
\hline
\end{tabular}

\subsubsection{Expërimentartion}

Le principe de la technique mise en oeuvre lors de ce traivail sonsis une messure ae l'évolutior des densitiés phiytopianctoniques au d'enceinmes immergées in situ er présence (enceintes expérimeriales) absence (enceimtes témoins) de zooplancton.

Les enceintes utilisées, construites en plexigias, sort de forme cubir d'une capacité de $3,375 \mathrm{l}$. Sur cinq de leurs faces sont prlacées, dans un de $13 \mathrm{~cm}$ de ciiamètre, des membranes filtrantes Nuclépores de $4 \mu$ porosité, permettant un rencuuvellement cie l'eau. Sur la sixiième face sidpérieure) somt placés les dispositifis de fixation de l'enceinte sur te câl soutien alinsi que le système de remplissagre et de vidanıje.

Des mesuress de vitesse de difiusion d'un colorant (Frose Erengel au $t$ | de ces membrianes, nous cont permis d'évaluer à 20 heures le tem renouvelliement complext de l'erau danss nos enceintes.

Les mesuress du troutaģe ont èté réialisées en Lin point central retenue ( $38 \mathrm{~m}$ cre profcindeur).

\section{a) Expérience du 20 maars $19 \mathrm{~g}: 2$}

Pour shaque profondeur de mesure. à savoir 5 et $75 \mathrm{~m}$, deux ence une experimentiale et Lune témoin. orit été untilisées. Elles ont été remplie: ¿ı l'eal prélevvée ah:x profrondsurs retennues. Pour l'enceninte témc z:oplaniston esi: préaiablement 
porosité. Parallèlement, $100 \mathrm{ml}$ d'eau de chacune des enceintes sont prélevés et additionnés de lugol pour dénombrement ultérieur, sous microscope inversé, des densités phytoplanctoniques au temps initial.

Les enceintes sont ensuite immergées à la profondeur d'origine de l'eau qu'elles contiennent et laissées en incubation pendant 8 heures (de $10 \mathrm{~h}$ à $18 \mathrm{~h}$ ). En fin d'expérience, $100 \mathrm{ml}$ d'eau de chacune des enceintes sont prélevés, additionnés de lugol, pour dénombrement des espèces phytoplanctoniques. La densité algale étant particulièrement faible lors de la réalisation de nos expériences, les dénombrements réatisés au microscope inversé ont été effectués sur un nombre derchamps optiques variables selon les espèces (de 30 à 4570 , soit la totalité de la chambre de sédimentation) et les grossissements utilisés. Le peuplement zooplanctonique présent dans l'enceinte expérimentale est récolté par filtration sur nylon de $30 \mu \mathrm{m}$ de porosité et immédiatement fixé au formol $4 \%$. Les dénombrements ont été réalisés en cuve de DOLFFUS après coloration au Rose Bengal. Les effectifs spécifiques ont été convertis en terme de biomasse à partir des relations longueur-poids établies par DUMONT et al. (1975).

\section{b) Expériences des 27 et 28 mai 1992}

La démarche utilisée lors de ces expérimentations est similaire à celle décrite précédemment. La seule différence réside dans le fait que les enceintes expérimentales contiennent de l'eau de la profondeur de mesure, préalablement filtrée comme celle des enceintes témoins, et additionnée d'un effectif précis de Copépodes triés à partir d'un prélèvement in situ. Les densités de Copépodes, par espèce et par stade de développement, introduits dans les enceintes expérimentales, sont identiques à celles trouvées dans le lac 24 heures auparavant lors du cycle nycthéméral que nous avons réalisé la veille des expériences. La durée d'incubation est de huit heures aussi bien pour la mesure de broutage réalisée de jour ( $10 \mathrm{~h}$ à $18 \mathrm{~h})$ que pour celle réalisée de nuit ( $22 \mathrm{~h}$ à $6 \mathrm{~h}$ ).

Le calcul du taux horaire de prédation par unité de biomasse zooplanctonique a été réalisé à partir des hypothèses suivantes (MAYANS, 1987):

- au sein des enceintes, la croissance phytoplanctonique est de type exponentiel.

- à tout instant, la prédation s'effectue sur une fraction constante du peuplement algal.

- le taux de prédation est constant durant toute la période d'incubation.

Bien que l'eau soit soigneusement homogénéisée avant remplissage des enceintes, les densités phytoplanctoniques sont susceptibles d'être différentes au temps zéro entre enceintes témoins et expérimentales. Nous avons donc procédé aux dénombrements cellulaires dans chacune des enceintes afin de prendre en compte cette éventuelle hétérogénéité qui s'ajoute aux inévitables incertitudes liées aux dénombrements.

- Dans ces conditions, pour chaque population phytoplanctonique, le taux de croissance spécifique intrinsèque, (u) peut être calculé à partir des informations obtenues dans l'enceinte témoin selon la relation : 


$$
N T_{t}=N T_{0} \cdot e^{u t}
$$

où $\mathrm{NT}_{1}$ : nombre de cellules au temps $t$ dans l'enceinte témoin ;

$N T_{0}$ : nombre de cellules au temps $t_{0}$ dans la même enceinte ;

$t$ : durée de l'incubation (h).

d'où,

$$
u=\left(\log N T_{t}-\log N T_{0}\right) / t .
$$

Dans l'enceinte expérimentale, le taux de croissance réel (r), résultante de la croissance intrinsèque $(\omega)$ et-de la prédation $(p)$ est donné par :

$$
N E_{t}=N E_{0} \cdot e^{r t}
$$

d'où,

$$
r=\left(\log N E_{t}-\log N E_{0}\right) / t
$$

avec $N_{t}$ : nombre de cellules dans l'enceinte expérimentale au temps $t$.

$\mathrm{NE}_{0}$ : nombre de cellules dans la même enceinte en début d'incubation.

Or,

$$
\mathrm{p}=\mathbf{u}-\mathrm{r}
$$

donc,

$$
p=\left[\left(\log N T_{t}-\log N T_{0}\right)-\left(\log N E_{t}-\log N E_{0}\right)\right] / t
$$

La quantité totale de cellules ingérées pour chaque population est l'intégrale de la courbe représentant le nombre de cellules ingérées à chaque instant. II est possible d'estimer ce nombre par la relation :

$$
P=p\left[\left(\mathrm{NE}_{0}+\mathrm{NE}_{\mathrm{t}}\right) / 2\right] \cdot 1 / \mathrm{B}
$$

où $P=$ le taux de prédation moyen par unité de biomasse zooplanctonique $(B)$ et par unité de temps.

\section{3 - RÉSULTATS}

\subsection{Expérience du 20 mars 1992}

a) Caractéristiques abiotiques et biotiques de la retenue lors de l'expérimentation

Les profils verticaux de température, $\mathrm{pH}$ et teneurs en oxygène dissous (fig. 1) mettent en évidence une absence de stratification des eaux; ces paramètres présentent de plus des valeurs très voisines pour les deux profondeurs (5 et $15 \mathrm{~m}$ ) où nos enceintes ont été placées en incubation. La profondeur de disparition du disque de Secchi est de $5,60 \mathrm{~m}$; par conséquent, le niveau de compensation photique se situe à $15,20 \mathrm{~m}$ environ. Les résultats de la production primaire (fig. 2) indiquent une intensité photosynthétique maximale à la profondeur $2 \mathrm{~m}$. 
Figure 1 Profils verticaux de température, pH et teneurs en oxygène dissous le 20 mars 1992 (-) et le 27 mai $1992(---)$ au sein de la retenue Al Massira.

Distribution of temperature, $\mathrm{pH}$ and oxygen concentrations $\left(\mathrm{mg} \cdot \mathrm{r}^{1}\right)$ on $20 \mathrm{March}(\longrightarrow)$ and 27 May $1992(---)$ in the water column of Al Massira Reservoir.

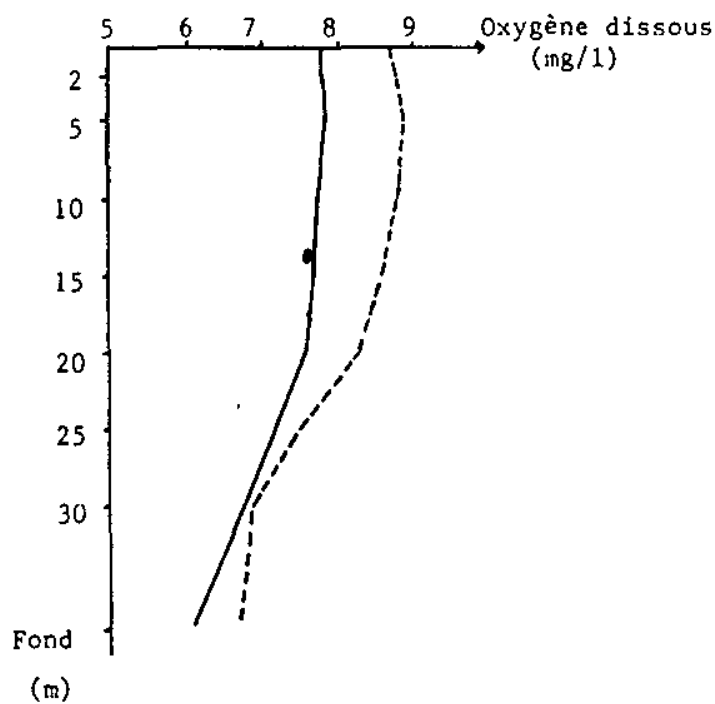

(m)

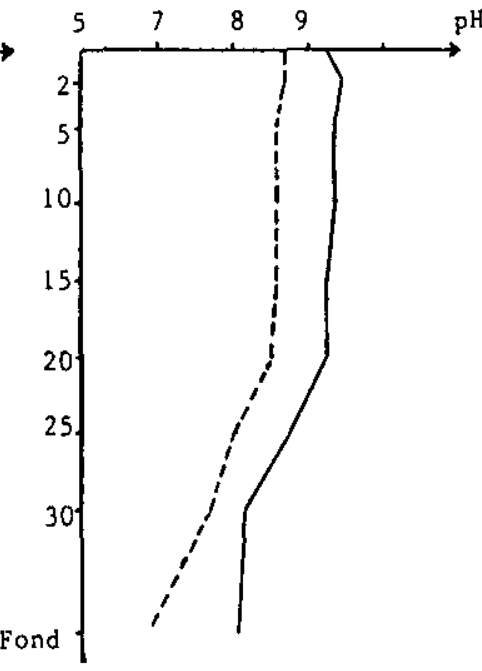

Profondeur (m)

(m)

water column of AlMassira Reservoir. 


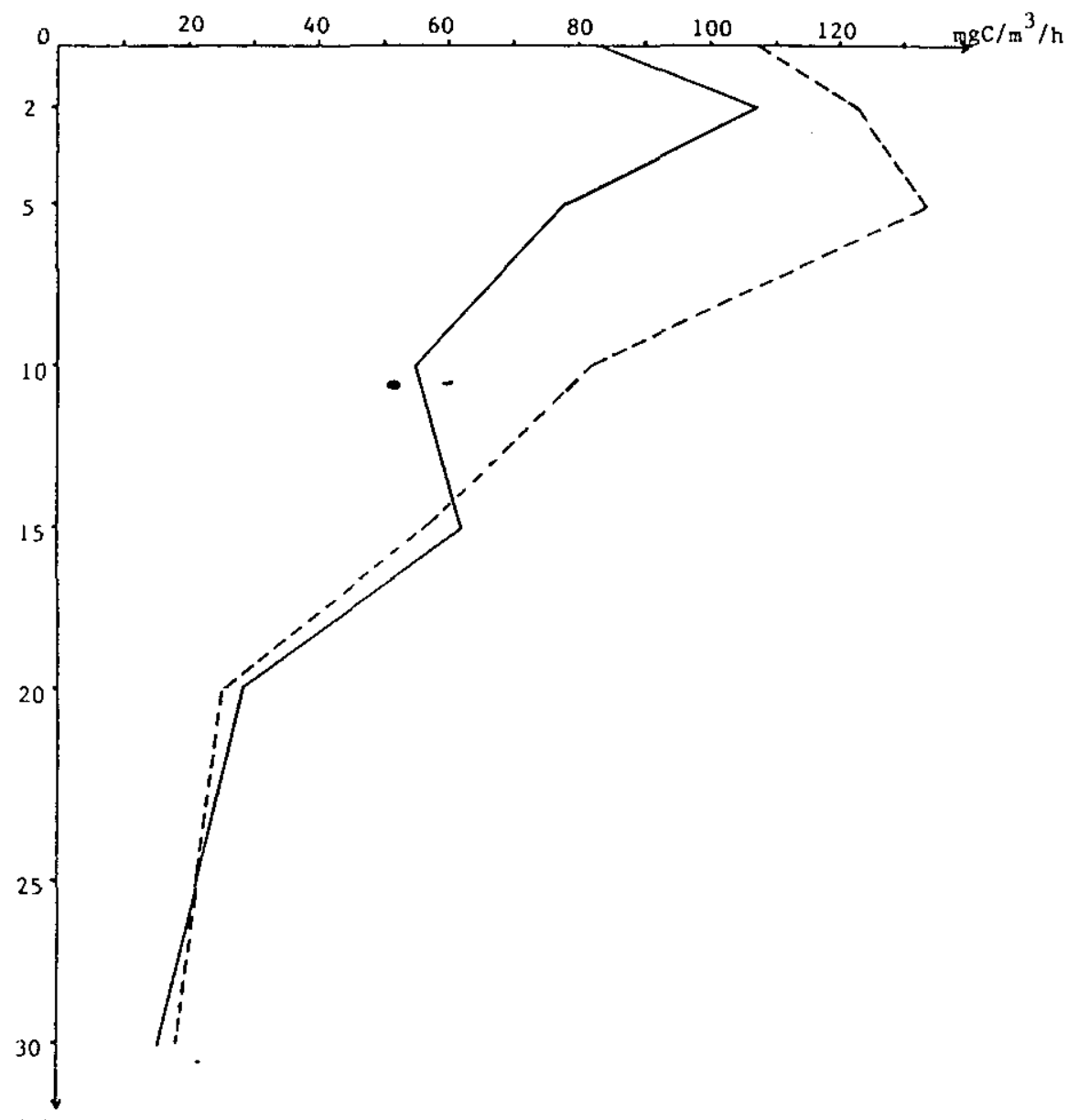

(m)

Figure 2 Profil vertical de la production primaire le 20 mars 1992 (-) et le 27 mai 1992 (- - ) au sein de la retenue Al Massira.

Changes in the primary production measured on 20 March (-) and 27 May $1992(--)$ in the water column of Al Massira Reservoir.

Le peuplement phytoplanctonique, qualitativement et quantitativement relativement homogène sur les vingt premiers mètres de la colonne d'eau, est essentiellement composé de Dinophycées (Peridinium cinctum, Ceratium hirundinella...), de Chlorophycées (Oocystis crassa, Scenedesmus ecornis...) et de Diatomées (Cyclotella sp.), les densités cellulaires étant très variables d'une espèce à l'autre (fig. 3).

La communauté zooplanctonique, dont la densité moyenne, déterminée par dénombrement sur eau brute, est de 46 individus par litre, est essentiellement composée de Copépodes $(50,4 \%$. de l'effectif total) et de Cladocères $(47,6 \%)$, les Rotifères ne constituant qu'une fraction minime (2\%) de l'ensemble. Du point de vue qualitatif, les Copépodes sont représentés par le Calanoïde Neolovenula alluaudi $(81,5 \%)$ et les 

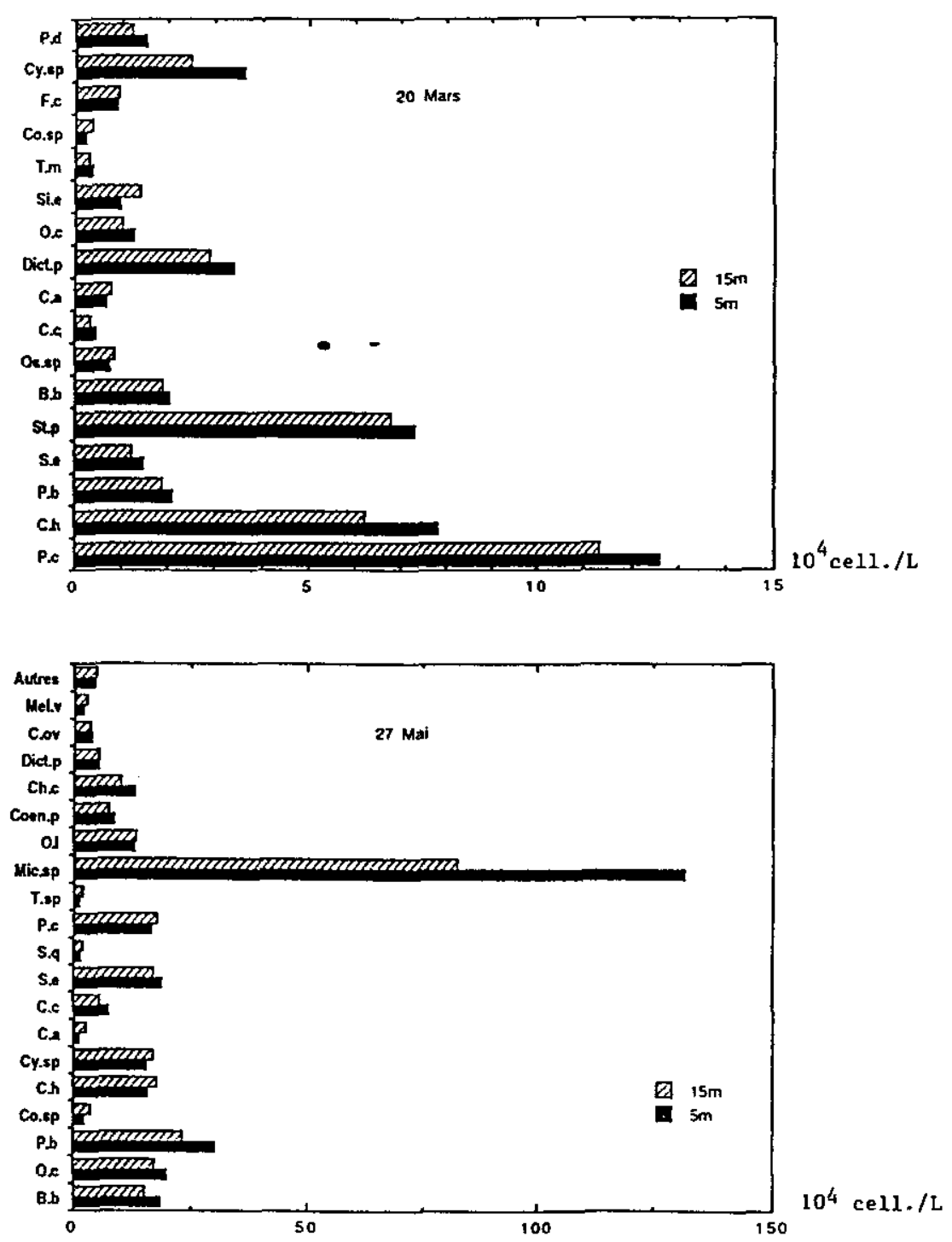

Figure 3 Densités des espèces phytoplanctoniques à 5 et à $15 \mathrm{~m}$ le 20 mars et le 27 mai 1992.

Distribution of phytoplankton species-numbers at 5 and $15 \mathrm{~m}$, on 20 and 27 May 1992.

P.c : Peridinium cinctum - C.h : Ceratium hirundinella - P.d : Pediastrum boryanum S.e : Scenedesmus ecornis - St.p : Staurastrum pingue - B.b : Botryococcus braunii Os.sp : Oscillatoria sp - C.c : Coelastrum cambricum - C.a : Closterium acicularae Dict.p : Dictyosphaerium pulchelum - 0.c : Oocystis crassa - Si.e : Surirella elegans T.m : Tetraedron minimum - Co.sp : Cosmanium sp - F.c : Fragilaria crotonensis - Cy.sp : Cy clotella $s p$ - P.d : Pediastrum duplex - Mel.v: Melosira varians - C.ov: Cryptomonas ovata Ch.c: Chroomonas caudata - Coen.p : Coenococcus planctonicus - 0.1 : Oocystis lacustris Mic.sp : Microcystis sp - T. sp : Tetraedron sp-S.q : Scenedesmus quadricauda. 
Cyclopoïdes Acanthocyclops robustus $(16,5 \%)$ et à un degré moindre Tropocyclops prasinus (2\%), les Cladocères par Diaphanosoma brachyurum (espèce dominante), Daphnia longispina et Ceriodaphnia reticulata.

Aux profondeurs d'expérimentation retenues (5 et $15 \mathrm{~m}$ ), les biomasses zooplanctoniques totales (poids sec) sont respectivement de 271 et $194 \mu \mathrm{g} / \mathrm{l}$.

b) Intensité de broutage

Considérée globalement, après incubation, l'évolution de la densité phytoplanctonique au sein des enceintes apparaît cohérente.

Dans les enceintes témoins, isolé de toute pression de prédation, le peuplement phytoplanctonique global voit ses effectifs augmenter beaucoup plus sensiblement $(9,9 \%)$ à $5 \mathrm{~m}$ qu'à $15 \mathrm{~m}(1,2 \%)$ où l'atténuation lumineuse limite très vraisemblablement l'activité photosynthétique (fig. 4).

Dans les enceintes expérimentales, les effectifs diminuent de $9 \%$ à $5 \mathrm{~m}$ et de $7,4 \%$ à $15 \mathrm{~m}$. Si ces deux valeurs relatives peuvent sembler comparables, il n'en va pas de même lorsque l'on considère le nombre de cellule consommées par unités de temps et de biomasse zooplanctonique: 43 cell. $/ \mu \mathrm{g} \cdot \mathrm{h}$ à $5 \mathrm{~m}$ et $24 \mathrm{cell} . / \mu \mathrm{g} \cdot \mathrm{h}$ à $15 \mathrm{~m}$. Ceci traduit le fait qu'à $5 \mathrm{~m}$, une part importante des cellules consommées est constituée de cellules produites lors de l'incubation, cette production étant très nettement plus importante à $5 \mathrm{~m}$ qu'à $15 \mathrm{~m}$ (fig. 2).

La seule analyse de l'évolution quantitative du peuplement phytoplanctonique global masque en fait d'importantes différences entre les principales espèces présentes (fig. 3 et 4). Si l'on considère que la précision des dénombrements cellulaires n'excède pas 5 à $10 \%$ selon les espèces, en raison de la faible densité lors de notre expérience, six espèces seulement présentent des variations significatives d'effectifs (fig. 4 et 5). II s'agit des Chlorophycées Scenedesmus ecornis, Coelastrum cambricum, Oocystis crassa, Tetraedron minimum, Cosmarium sp. et de la Diatomée Cyclotella sp. En nombre de cellules consommées, c'est incontestablement Cyclotella qui supporte la plus importante pression de prédation (fig. 4 et 5).

\subsection{Expériences du 27 et 28 mai 1993}

a) Caractéristiques abiotiques et biotiques de la retenue lors des expérimentations

Tout comme lors de l'expérience précédente, les profils verticaux de température, $\mathrm{pH}$ et teneurs en oxygène dissous, mettent en évidence une absence de stratification des eaux (fig. 2). De plus, ces paramètres présentent des valeurs très voisines pour les deux profondeurs d'expérimentation (5 et $15 \mathrm{~m})$. La profondeur de disparition du disque de Secchi $(4,80 \mathrm{~m})$ est par contre inférieure à la valeur trouvée le 20 mars. Le profil vertical de la production primaire indique un maximum d'activité photosynthétique à $5 \mathrm{~m}$. 


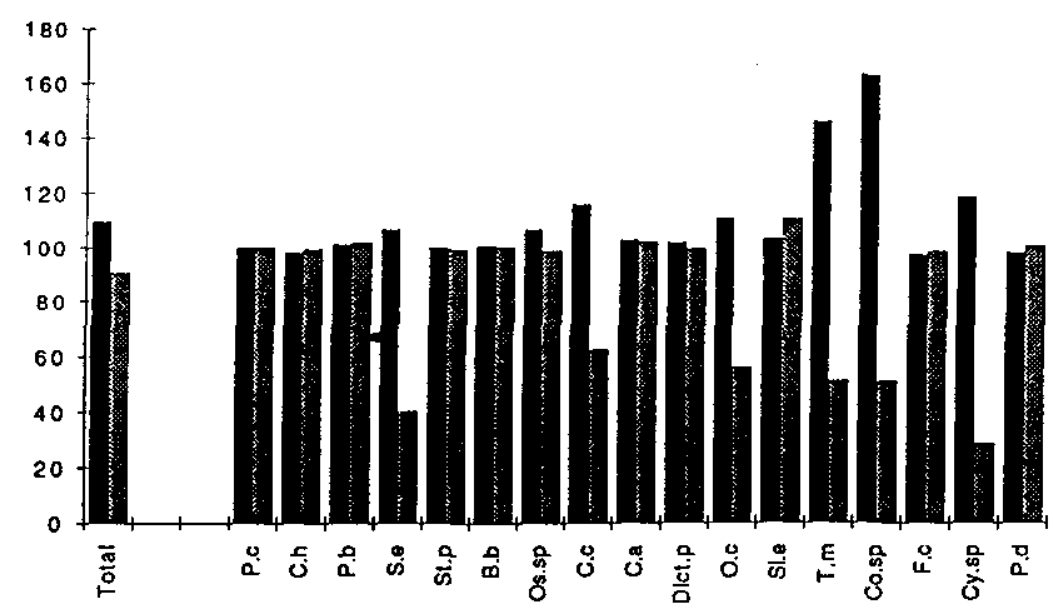

$15 \mathrm{~m}$

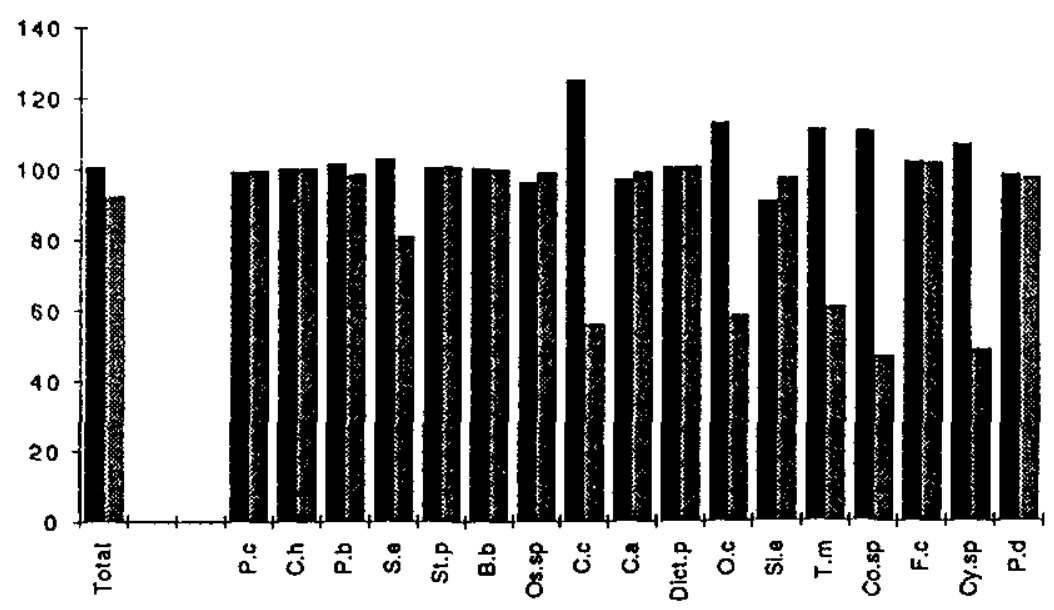

Figure 4 Évolution de la densité du peuplement phytoplanctonique global et des principales composantes (en \% de $\mathrm{Nt}_{0}$ ) en absence (noir) et en présence (gris) de zooplancton après 8 heures d'incubation (20 mars 1992).

Changes in the total phytoplankton cell numbers $\left(\mathrm{Nt}_{\sigma}\right)$ and in the principal taxa percents (as \% of $\mathrm{Nt}_{0}$ ) when the flasks were free of (black) or contained (grey) copepods over 8 hours of incubation, on 27 May 1992.

P.c : Peridinium cinctum - C.h : Ceratium hinundinella - P.d : Pediastrum boryanumS.e : Scenedesmus ecornis - St.p: Staurastrum pingue - B.b : Botryococcus braunii Os.sp : Oscillatoria $s p-$ C.c : Coelastrum cambricum - C.a : Closterium acicularae Dict.p : Dictyosphaerium pulchelum - 0.c : Oocystis crassa - Si.e : Surirella elegans T.m : Tetraedron minimum - Co.sp : Cosmanium sp-F.c : Fragilaria crotonensis - Cy.sp : Cy clotella sp - P.d: Pediastrum duplex-Mel.v: Melosira varians - C.ov: Cryptomonas ovata Ch.c : Chroomonas caudata - Coen.p : Coenococcus planctonicus - 0.1 : Oocystis lacustris Mic.sp : Microcystis $s p-$ T. $s p$ : Tetraedron $s p-\mathrm{S} . q$ : Scenedesmus quadricauda. 


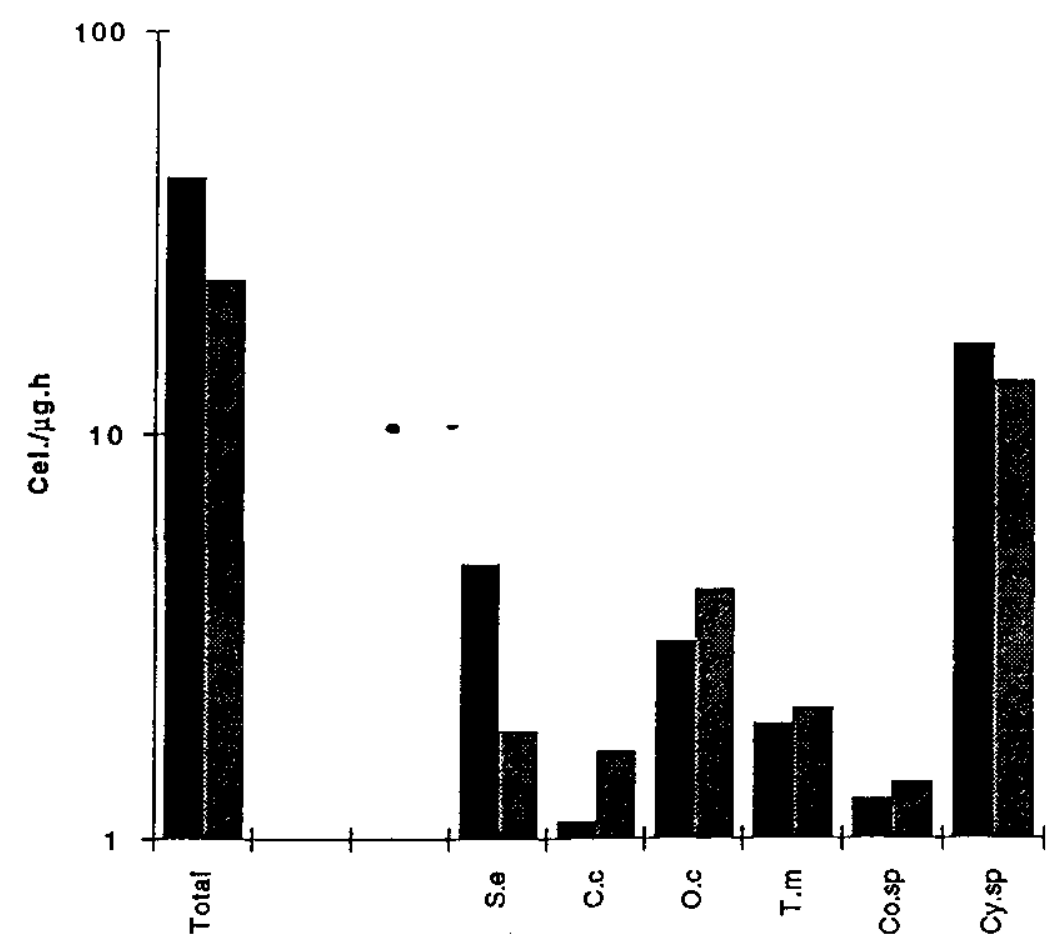

Figure 5 Intensité du broutage du peuplement phytoplanctonique par le zooplancton à $5 \mathrm{~m}$ (noir) et à $15 \mathrm{~m}$ (gris) (20 mars 1992).

Intensity of the grazing pressure exerted by zooplankton on algae at $5 \mathrm{~m}$ (black) and at $15 \mathrm{~m}$ (grey), on 20 March 1992.

S.e : Scenedesmus ecornis - C.c : Coelastrum cambricum-0.c: Oocystis crassaT.m: Tetraedron minimum - Co.sp : Cosmanium sp-Cy.sp : Cyclotella sp .

Le peuplement phytoplanctonique, plus diversifié que lors de la première expérience, est quantitativement dominé par (fig. 3) des Dinophycées (Peridinium cinctum, Ceratium hirundinella..), des Cyanophycées (Microcystis sp.), des Chlorophycées (Oocystis crassa, Scenedesmus ecornis.), des Diatomées (Cyclotella sp.) et des Cryptophycées (Chroomonas caudata et Cryptomonas ovata).

Le peuplement de Copépodes, représentant plus de $90 \%$ de la biomasse totale du zooplancton présent, est toujours quantitativement dominé par Neolovenula alluaudi (18 ind/l) suivi d'Acanthocyclops robustus (12 ind//) et à un degré moindre de Tropocyclops prasinus ( 3 ind/l). Les résultats d'un cycle nycthéméral (tableau 2), réalisé 24 heures avant nos expériences de broutage, indiquent que les stades de développement des différents Copépodes de la retenue Al Massira accomplissent d'importantes migrations verticales entre le jour et la nuit (MONCEF, à paraître). 
Tableau 2 Pourcentages d'effectifs des nauplius, copépodites et adultes à 5 et à $15 \mathrm{~m}$ respectivement le jour et la nuit le 27 et 28 mai 1992.

Table 2 Percentage of numbers of nauplii, copepodites and adult zooplankton species at 5 and $15 \mathrm{~m}$, respectively over the day and the night on 27 and 28 May 1992.

\begin{tabular}{|lcccc|}
\hline & $\mathbf{5} \mathbf{m}$ (jour) & $\mathbf{5} \mathbf{m}$ (nujit) & $\mathbf{1 5} \mathbf{m}$ (jour) & $\mathbf{1 5} \mathbf{m}$ (nuit) \\
\hline Nauplius & 47,6 & 40,2 & 45,6 & 33,4 \\
Copépodites & 21,9 & 22,3 & 20,3 & 26,6 \\
Adultes & 30,5 & 37,5 & 34,1 & 40 \\
\hline
\end{tabular}

b) Intensité du broutage

b1) Broutage diurne

Les biomasses de Copépodes contenus dans les enceintes expérimentales à 5 et à $15 \mathrm{~m}$, sont respectivement de 1004 et $681 \mu \mathrm{g} / \mathrm{l}$ (poids sec).

Au sein des enceintes témoins, en dehors de toute activité de broutage, la croissance phytoplanctonique (fig. 6) est sensiblement plus élevée à $5 \mathrm{~m}$ $(6,8 \%)$ qu'à $15 \mathrm{~m}(3,1 \%)$.

Dans les enceintes expérimentales, les densités algales chutent de $9,2 \%$. à $5 \mathrm{~m}$ et $4,7 \%$ à $15 \mathrm{~m}$ entre le début et la fin de l'incubation. Par ailleurs, le nombre de cellules ingérées par unité de biomasse zooplanctonique et de temps (fig. 7), est plus important à $5 \mathrm{~m}(63 \mathrm{celf} . / \mu \mathrm{g} \cdot \mathrm{h})$ qu'à $15 \mathrm{~m}(39,7 \mathrm{cell} . / \mu \mathrm{g}$ - h). Ainsi l'intensité du broutage exercé par les Copépodes est beaucoup plus élevée à la profondeur où la croissance algale est la plus importante.

L'évolution quantitative du peuplement phytoplanctonique total (fig. 6) indique que seules dix espèces présentent des variations d'effectifs. II s'agit des Chlorophycées Scenedesmus ecornis, Coelastrum cambricum, Oocystis crassa, Scenedesmus quadricauda, Tetraedron minimum, Cosmarium sp. et Oocystis lacustris, de la Diatomée Cyclotella sp. et des Cryptophycées Chroomonas caudata et Cryptomonas ovata. En nombre de cellules consommées, il est clair que c'est Cyclotella sp. qui subit, encore une fois, la plus forte activité de broutage aussi bien à 5 qu'à $15 \mathrm{~m}$ (fig. 7 ).

\section{b2) Broutage nocturne}

Les biomasses (exprimées en poids sec) des Copépodes introduits au sein les enceintes expérimentales sont respectivement de $1142 \mu \mathrm{g} / \mathrm{l}$ à $5 \mathrm{~m}$ et $j 90 \mu \mathrm{g} / \mathrm{l}$ à $15 \mathrm{~m}$.

Les résultats obtenus (fig. 8 et 9) mettent en évidence ;

- d'une part une croissance algale nulle dans les enceintes témoins aux deux profondeurs d'expérimentation, ce qui est évidement lié à l'absence d'énergie lumineuse, mais conforte la validité des dénombrements cellulaires, donc de notre méthode expérimentale,

- d'autre part, au sein des enceintes expérimentales, il y a diminution des effectifs phytoplanctoniques de $14,6 \%$ à $5 \mathrm{~m}$ et de $4,2 \%$ seulement à $15 \mathrm{~m}$ (fig. 9). En terme de nombre de cellules ingérées, ceci correspond à 42,3 cell. $/ \mu \mathrm{g} \cdot \mathrm{h}$ à $5 \mathrm{~m}$ et 16,4 cell. $/ \mu \mathrm{g} \cdot \mathrm{h}$ à $15 \mathrm{~m}$ (fig. 8 ). 


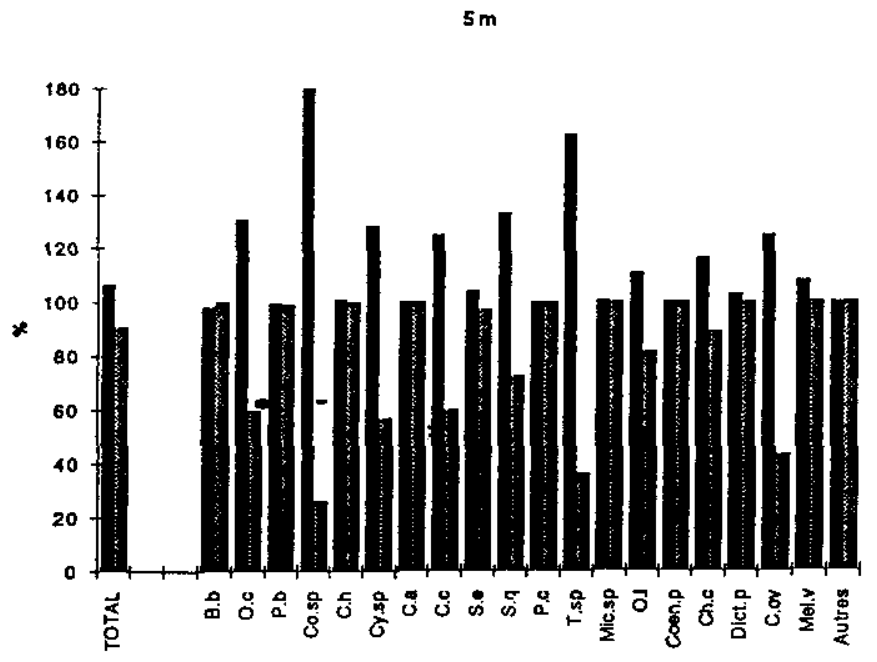

$15 \mathrm{~m}$

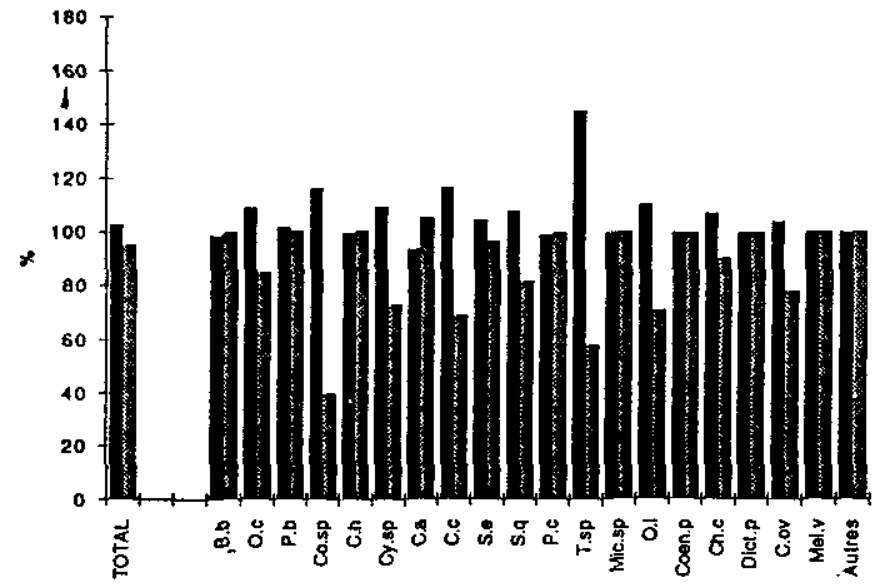

Figure 6 Broutage diurne des copépodes - Évolution de la densité du peuplement phytoplanctonique global et des principales composantes (en \% de $\mathrm{Nt}_{0}$ ) en absence (noir) et en présence (gris) de copépodes après 8 heures d'incubation (27 mai 1992).

Copepod day-grazing: changes in the total phytoplankton cell numbers ( $N t_{\alpha}$ ) and in the principal taxa percents (as \% of $N t_{\partial}$ ) when the flasks were free of (black) or contained (grey) copepods over 8 hours of incubation, on 27 May 1992.

P.C: Peridinium cinctum - C.h : Ceratium hirundinella - P.d : Pediastrum boryanum S.e : Scenedesmus ecornis - St.p : Staurastrum pingue - B.b : Botryococcus braunit Os.sp : Oscillatoria sp-C.c : Coelastrum cambricum-C.a : Closterium acicularae Dict.p : Dictyosphaerium pulchelum - 0.c : Oocystis crassa - Si.e : Surirella elegans T.m : Tetraedron minimum - Co.sp : Cosmarium sp - F.c : Fragilaria crotonensis - Cy.sp : $C y$ clotella s $\rho$ - P.d: Pediastrum duplex - Mel.v: Melosira varians - C.ov: Cryptomonas ovataCh.c: Chroomonas caudata - Coen.p : Coenococcus planctonicus - 0.1: Oocystis lacustris Mic.sp : Microcystis sp - T. sp : Tetraedron sp - S.q : Scenedesmus quadricauda. 


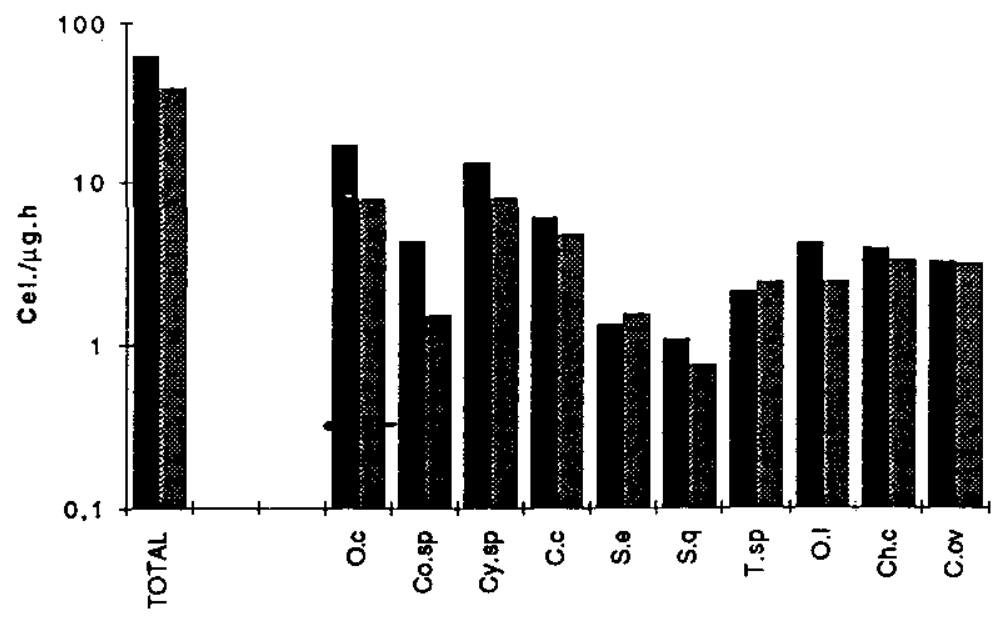

Figure 7 Intensité du broutage diurne du peuplement phytoplanctonique par les copépodes à $5 \mathrm{~m}$ (noir) et à $15 \mathrm{~m}$ (gris) (27 mai 1992).

Intensity of the day-grazing pressure exerted by copepods on algae at $5 \mathrm{~m}$ (black) and at $15 \mathrm{~m}$ (grey), on 27 May 1992).

0.c : Oocystis crassa - Co.sp : Cosmarium sp-Cy.sp : Cyclotella sp - C.c : Coelastrum cam bricum-S.e : Scenedesmus ecomis - S.q: Scenedesmus quadricauda - T. sp : Tetraedron sp 0.1: Oocystis lacustris - Ch.c: Chroomonas caudato - C.ov: Cryptomonas ovata.

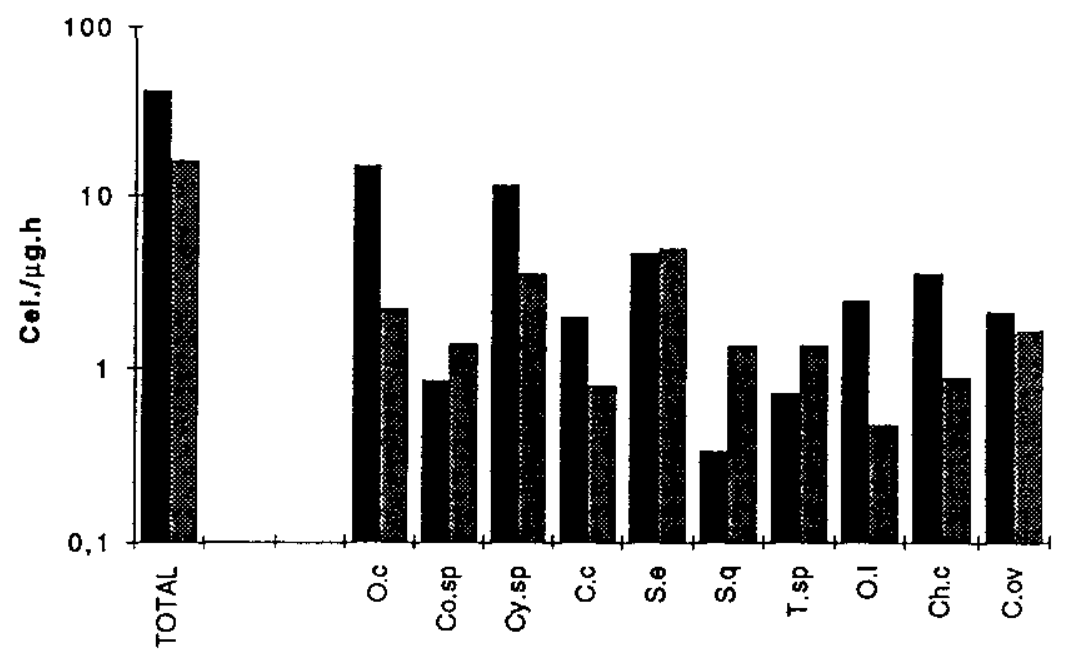

Figure 8 Intensité du broutage nocturne du peuplement phytoplanctonique par les copépodes à $5 \mathrm{~m}$ (noir) et à $15 \mathrm{~m}$ (gris) (27-28 mai 1992).

Intensity of the day-grazing pressure exerted by copepods on algae at $5 \mathrm{~m}$ (black) and at $15 \mathrm{~m}$ (grey), on 27-28 May 1992).

$0 . c$ : Oocystis crassa - Co.sp : Cosmarium $s p-C y . s p$ : Cyclotella $s p-C . c$ : Coelastrum cam bricum-S.e : Scenedesmus ecomis - S.q : Scenedesmus quadricauda -T. $\mathrm{sp}:$ Tetraedron $s p$ 0.1: Oocystis lacustris - Ch.c: Chroomonas caudata - C.ov: Cryptomonas ovata. 
$5 \mathrm{~m}$

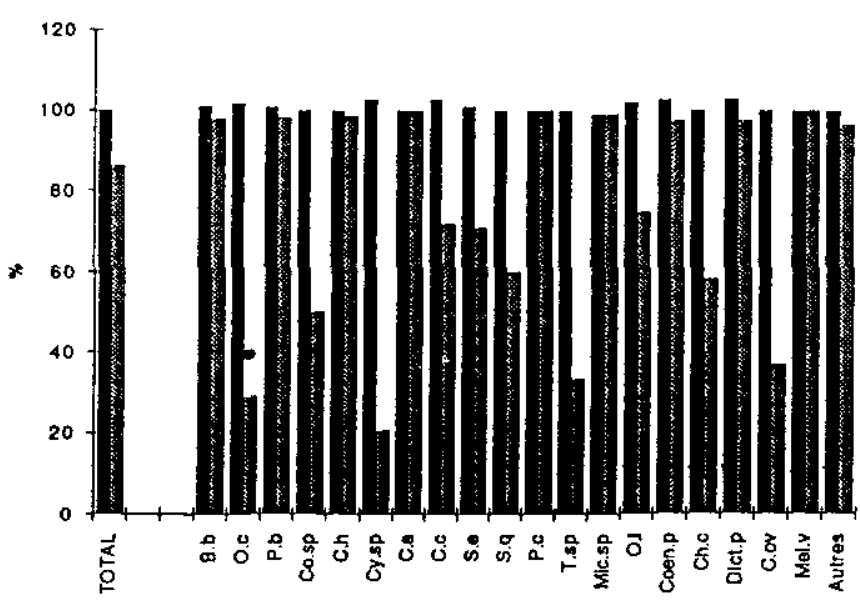

$15 \mathrm{~m}$

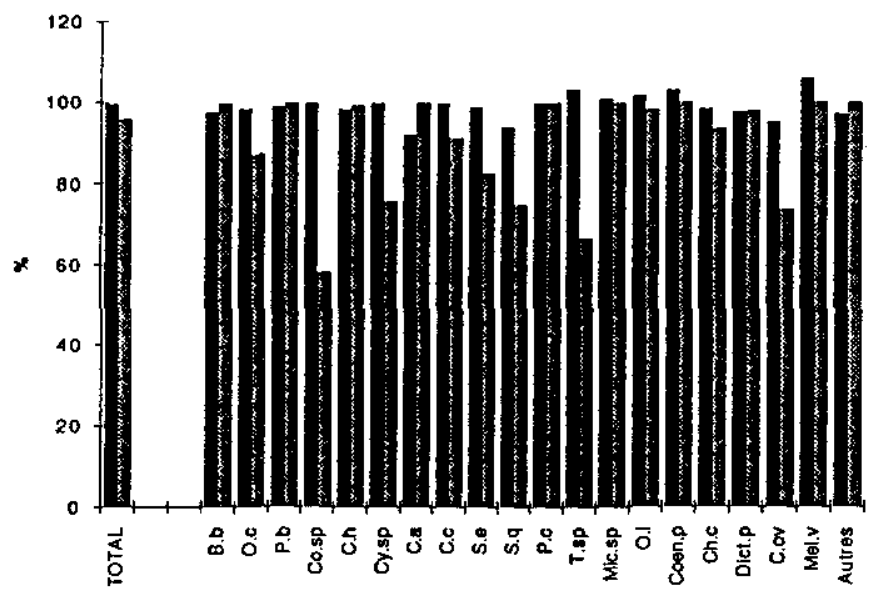

Figure 9 Broutage nocturne des copépodes - Évolution de la densité du peuplement phytoplanctonique global et des principales composantes (en \% de $\mathrm{Nt}_{0}$ ) en absence (noir) et en présence (gris) de copépodes après 8 heures d'incubation (27-28 mai 1992).

Copepod day-grazing: changes in the total phytoplankton cell numbers ( $N t_{\alpha}$ ) and in the principal taxa percents (as \% of $N t_{\alpha}$ ) when the flasks were free of (black) or contained (grey) copepods over 8 hours of incubation, on 27-28 May 1992.

P.c : Peridinium cinctum - C.h : Ceratium hirundinella - P.d: Pediastrum boryanum S.e : Scenedesmus ecornis - St.p: Staurastrum pingue - B.b : Botryococcus braunii Os.sp : Oscillatoria $s p-C . c:$ Coelastrum cambricum - C.a : Closterium acicularaeDict.p : Dictyosphaerium pulchelum - 0.c: Oocystis crassa - Si.e : Surirella elegans T.m : Tetraedron minimum - Co.sp : Cosmarium sp-F.c : Fragilaria crotonensis - Cy.sp : Cy clotella $s p-P . d$ : Pediastrum duplex - Mel.v: Melosira varians - C.ov : Cryptomonas ovataCh.c : Chroomonas caudata - Coen.p : Coenococcus planctonicus - 0.1 : Oocystis lacustris Mic.sp : Microcystis $s p-$ T. $s p$ : Tetraedron $s p-S . q$ : Scenedesmus quadricauda. 
L'analyse quantitative par espèce algale (fig. 9), nous permet de remarquer qu'en dehors des dix espèces précédemment citées, aucune ne présente des variations de densité entre le début et la fin de l'expérience. L'intensité du broutage est, par ailleurs, plus faible à $15 \mathrm{~m}$ qu'à $5 \mathrm{~m}$ (fig. 8), et la plus forte pression de prédation s'exerce sur la Chlorophycée Oocystis crassa $(15,4$ cell. $/ \mu \mathrm{g} \cdot \mathrm{h})$ à $5 \mathrm{~m}$ et de nouveau la Diatomée Cyclotella $s p(11,8 \mathrm{cell} . / \mu \mathrm{g} \cdot \mathrm{h})$ à $15 \mathrm{~m}$.

\section{4 - DISCUSSION ET CONCLUSIONS}

Les résultats de nos expériences sont tout à fait conformes au données de la littérature (BOGDAN et MG NAUGHT, 1975; GAWLER et ANGELI, 1987 ; BORSHEIM et ANDERSON, 1987...), dans la mesure où le broutage apparaît bien comme sélectif, plusieurs espèces algales n'étant pas consommées.

\subsection{Critères de sélection des algues}

Deux variables semblent influencer la sélectivité du broutage :

- la dimension des cellules. En effet, seules les algues de petite taille présentent des variations importantes d'effectifs dans les enceintes expérimentales entre le temps initial et le temps final de l'expérience aussi bien lors du broutage diurne que nocturne. D'autres espèces même abondantes, mais de grande taille (Peridinium cinctum, Ceratium hirundinella et Staurastrum pingue), ne montrent aucune variation de densité.

- une éventuelle détection des proies par le biais des métabolites excrétés par les cellules algales physiologiquement actives, dans la mesure où seules les petites espèces présentant un développement important au sein des enceintes témoins sont soumises à la prédation.

\subsection{Sélection au cours du broutage diurne}

Si au sein du peuplement phytoplanctonique global, le développement algal dans les enceintes témoins paraît peu important en valeurs relatives $(6,8 \%$ à $5 \mathrm{~m}$ et $3,1 \%$ à $15 \mathrm{~m})$, il n'en va pas de même lorsque nous analysons l'évolution des effectifs de chaque espèce. En effet, en fin d'expérience, les augmentations d'effectifs au sein des enceintes témoins atteignent $78 \%$ de la densité initiale chez Cosmarium sp., et $73 \%$ à $5 \mathrm{~m}$ puis $39 \%$ a $15 \mathrm{~m}$ chez Tetraedron $\mathrm{sp}$.

D'autre part, seules les espèces présentant à la fois une petite taille et un développement important, sont préférentiellement sélectionnées par les Copépodes présents. Les autres ne subissent pratiquement aucune variation aussi bien dans les enceintes témoins qu'expérimentales. De plus, la présente 
expérience confirme le fait que la dimension des cellules ne constitue pas le seul critère de sélection des proies. En effet, nous constatons que :

- les plus fortes pressions de prédation s'exercent particulièrement sur les espèces phytoplanctoniques à forte croissance (Cosmarium sp. et Tetraedron $s p$.$) ; la détection de ces algues pouvant se faire par le biais des métabolites$ excrétés.

- certaines espèces telles que Cœlastrum cambricum et Cryptomonas ovata (fig. 6) ne subissent pas la même pression de prédation bien que leurs développements soient similaires au sein des enceintes témoins à $5 \mathrm{~m}$. Ceci serait probablement lié aux préférences des copépodes vis-à-vis des algues consommables disponibles,(DEMOTT, 1986).

\subsection{Sélection au cours du broutage nocturne}

Le broutage demeure important et porte sur les mêmes espèces algales que durant le jour. Ceci incite à penser que la détection des proies se fait également à partir de métabolites non issus de l'activité photosynthétique.

Seules les espèces de petite taille sont consommées. La sélection est donc basée en grande partie, sur la dimension des cellules.

Conformément aux données rapportées dans la littérature, la taille des cellules phytoplanctoniques apparaît bien, en raison des capacités mécaniques de capture des proies, comme l'un des critères majeurs de sélection par les crustacés planctoniques. Toutefois, dans le cas des copépodes plus spécialement étudiés ici, l'intensité de la pression de prédation semble très sélective, vraisemblablement associée à une détection chimique des cellules ingérées à partir de métabolites excrétés par les algues, cette excrétion n'étant pas uniquement induite par l'activité photosynthétique.

A $15 \mathrm{~m}$, l'intensité du broutage nocturne chute énormément pour une biomasse de copépodes très comparable à celle des expériences concernant le broutage diurne. Ceci serait lié à :

- la variation à cette même profondeur, des densités relatives des différents stades de développement des copépodes présents, entre le jour et la nuit (tableau 2). la nuit.

- une baisse probable des potentialités de détection des algues pendant

A $5 \mathrm{~m}$, malgré l'existence d'une biomasse de copépodes plus importante la nuit que le jour dans l'enceinte expérimentale, nous constatons une diminution de la prédation pendant la nuit, ce qui serait lié, comme c'était le cas à $15 \mathrm{~m}$, à un rythme nycthéméral de broutage probablement dû à une baisse de détection des espèces phytoplanctoniques.

A la suite de HANEY (1971), de nombreux auteurs (SINGH, 1972 ; MACKAS et BOHRER, 1976) ont conclu à l'existence d'un rythme nycthéméral d'activité de broutage, l'intensité maximale étant observée durant la nuit.

Nos résultats aboutissant à une conclusion opposée, deux hypothèses peuvent être émises :

- Lors de nos expériences, le peuplement de la retenue Al Massira était quantitativement dominé par le Calanoïde Neolovenula alluaudi. Or, selon 
HANEY et HALL (1975), ces copépodes présentent une variabilité nycthémérale de leur activité de broutage relativement faible et, en tout état de cause, bien inférieure à celle des cladocères. II est donc possible, ce qui pourra être confirmé par des études ultérieures, que la variation enregistrée ici ne soit pas statistiquement significative et incluse dans la variabilité inhérente à tout matériel biologique.

- La méthode initiée par HANEY (1971) ne fournit qu'une mesure du volume d'eau transitant dans le tube digestif du zooplancton par unité de temps. Or dans la plupart des cas, ces expériences ont été réalisées au sein de lacs naturels présentant une stratification thermique marquée, c'est-à-dire une importante différence de température entre les eaux de surface (chaudes) et les eaux profondes (froides). Pour de simples raisons physiologiques, il est évident que dans les eaux profondes, les organismes présentent un métabolisme ralenti en raison de la température ambiante, ce métabolisme s'accélérant lors de la remonté nocturne au sein des couches superficielles (MC LAREN, 1963). En conséquence, il est évident que l'accélération de l'activité physiologique se traduit par une augmentation du volume d'eau transitant par le tube digestif des crustacés mais non fatalement par celle de la biomasse phytoplanctonique réellement digérée. Nos résultats, démontrant une importante sélectivité du broutage par les copépodes, vont plutôt dans le sens de cette interprétation, le choix de la nourriture excluant toute proportionnalité entre volume d'eau ingérée et apport énergétique.

\section{REMERCIEMENTS}

Nous remercions vivement M. R. POURRIOT pour sa fructueuse collaboration dans la rédaction de cet article, et $M$. D. PONT pour nous avoir fourni la technique d'expérimentation.

Nos remerciements s'adressent également à M. L. ALEYA pour la traduction du résumé, et à $M$. D. SARGOS pour son aide dans la réalisation des graphiques.

\section{RÉFÉRENCES BIBLIOGRAPHIQUES}

BOGDAN K.G., MCNAUGHT D.C., 1975. Selective feeding by Diaptomus and by Daphnia. Verh. Internat. Verein. Limnol. 19, 2935-2942.

BOURDIER G., AMBLARD C., 1989. Lipids in Acanthodiaptomus denticornis during starvation and fed on three different algae. J. Plankton Res., 11, 6, 1201-1212.

BOURDIER G., 1989. Composition biochimique du matériel particulaire lacustre: intérêt pour l'étude de l'activité métabolique des organismes, de la dynamique des 
populations phytoplanctoniques et des relations trophiques phyto-zooplancton. Thèse Doct., Université Clermont II, 292 pp.

DEMOTT $W ., 1986$. The role of taste in food selection by freshwater zooplankton. Oecologia. 69, 334-340.

DUMONT H.J., VAN DE VELDE I., DUMONT S., 1975. The dry weight estimate of biomass in a selection of Cladocera, Copepoda and Rotifera from the plankton, periphyton and benthos of confinental waters. Oecologia (Berf.) 19, 75-97.

GAWLER M., ANGELI N., 1987. Intensité du broutage dans le lac Léman en relation avec la structure de la taille des particules : variations nycthémérales. Natural. Can. : 114.

HANEY J.F., 1971. An in situ method for measurement of zooplancton grazing rates. Limnol. Oceanogr., 16, 970-977.

HANEY J.F., 1973. An in situ examination of the grazing activities of natural zooplankton communities. Arch. Hydrobiol., 72, 87-132.

HANEY J.F, HALL D.J., (1975). Diel vertical migration and filter-feeding activities of Daphnia. Arch. Hydrobiol., 75, 413-441.

MACKAS D., BOHRER R. (1976). Fluoroescence analysis of zooplankton gut contents and investigation of diel feeding pattems. J. Exp. Mar. Biol. Ecol., 77-85.

MALKł M., 1986. Contribution à l'étude de la communauté phytoplanctonique d'un écosystème lacustre marocain: Dayet ERROUmi. Thèse Doct. 3ème cycle, Rabat, 185 pp.
MAYANS M. Etude expérimentale in situ du broutage par te zooplancton (Daphnia longispina) sur le peuplement algal printanier d'une retenue oligotrophe (Ste Croix, Provence). DEA Université AixMarseille 1, $61 \mathrm{pp}$.

MC LAREN I.A., 1963. Effects of temperature on the growth of zooplankton and the adaptive value of vertical migration. J. Fish. Res. Baard Can., 26, 685-725.

MONCEF M., 1986. Étude de la communauté zooplanctonique d'un écosystème lacustre marocain : Dayet ERRoUMI. Thèse Doct. 3ème cycle, Rabat, 207 pp.

MOURELATOS S., POURRIOT R., ROUGIER C., 1990. Taux de filtration du Rotifére Brachionus calyciflorus : Comparaison des méthodes de mesure; Influence de l'âge. VIE MILIEU, 40 (1), 39-43.

MOURELATOS S., 1988. Broutage du phytoplancton par le zooplancton dans un lac peu profond. Thèse Doct., Université Paris 6, $191 \mathrm{pp}$.

PORTER K.G.,1972. A method for the in situ study of zooplankton grazing effects on species composition and standing crop. Limnol. Oceanogr., 17, 6, 913-977.

QUIBLIER G., BOURDIER G., AMBLARD G., PEPIN D., COMMUNAL P.Y., 1991. Intérêts de la séparation des pigments par C.L.H.P. pour l'étude des relations trophiques PhytoZooplancton. Rapport de la 3ème C.I. L.E.F., 104-107...

SINGH P.J., 1972. Studies on the food and feeding of the freshwater calanoid Rhinediaptomus indicus Kiefer $\|$ : Diurnal variations in feeding propensities. Hydrobiologia , 39, 209-215. 